\title{
Step structure of vicinal Ge(001) surfaces
}

\author{
Bart A.G. Kersten *, Harold J.W. Zandvliet, Dave H.A. Blank, A. van Silfhout \\ University of Twente, Department of Applied Physics, P.O. Box 217, 7500 AE Enschede, The Netherlands
}

Received 28 April 1994; accepted 21 September 1994

\begin{abstract}
The microscopic details of the step morphology of vicinal $\mathrm{Ge}(001)$ surfaces are analyzed on an atomic level using scanning tunnelling microscopy. Despite the strong correspondence to vicinal $\mathrm{Si}(001)$ surfaces, the step structure of the vicinal $\mathrm{Ge}(001)$ surface exhibits some significant differences. Rebonded, as wcll as nonbonded SB step cdges, are observed and, related to the occurrence of both configurations, kinks with a minimum length of $a_{0}(\approx 4 \AA)$ do occur. Kinks in SB rebonded step edges can be regarded as independent excitations whereas for kinks in SB nonbonded step edges this is definitely not the case. Occasionally dimers are found at the SB steps, which are located just in between two dimer rows of the upper terrace. Finally, for the first time a ferromagnetic-like coupling between buckled dimers in the same row is observed close to step edges.
\end{abstract}

Keywords: Equilibrium thermodynamics and statistical mechanics; Germanium; Scanning tunneling microscopy; Surface structure, morphology, roughness, and topography

\section{Introduction}

The morphology of vicinal surfaces, i.e. surfaces slightly misoriented with respect to one of their low Miller indices planes, is under continuous interest. The misorientation is, in the case of $\mathrm{Ge}(001)$ and $\mathrm{Si}(001)$ surfaces, accommodated by either single or double height steps depending on the exact miscut angle. The steps separate the adjacent terraces with the (001) orientation from each other. Step edges play an important role in phenomena such as crystal growth, surface chemistry, catalysis and the growth of epitaxial overlayers. Most attention, until now, is paid to the $\mathrm{Si}(001)$ surfaces with a small miscut towards the [011] direction $[1,2,4]$. The renewed interest in Ge surfaces is due mainly to the possibility to artificially create, using advanced crystal growth methods such as molecular beam epitaxy (MBE), heterostructures of alternating $\mathrm{Si}$ and Ge layers which exhibit unexpected new phys-

\footnotetext{
* Corresponding author.
}

ical phenomena. In this paper we will focus our attention on the important geometric and structural properties of the surface steps of germanium on an atomic scale.

Both, $\mathrm{Ge}(001)$ and $\mathrm{Si}(001)$ surfaces, reconstruct by forming dimers which arrange in parallel rows $[5,6]$. Depending on the crystal cut, either a $(2 \times 1)$ or a $(1 \times 2)$ reconstructed domain forms, related to each other by a $90^{\circ}$ rotation. If the surface has a slight miscut towards the [011] direction, single height steps are formed which separate terraces with alternating $(2 \times 1)$ or $(1 \times 2)$ reconstruction. In this configuration two types of steps, denoted by SA and SB, develop. The SA steps have the dimer rows of the upper terrace running parallel to the step edge, whereas for SB steps the dimer rows are oriented perpendicular to the edge itself.

Chadi [7] performed total-energy calculations on the SA and the SB steps of $\mathrm{Si}(001)$. For the SB step Chadi examined two possible configurations; arebonded and a non-bonded configuration (see Fig. 1). 
a

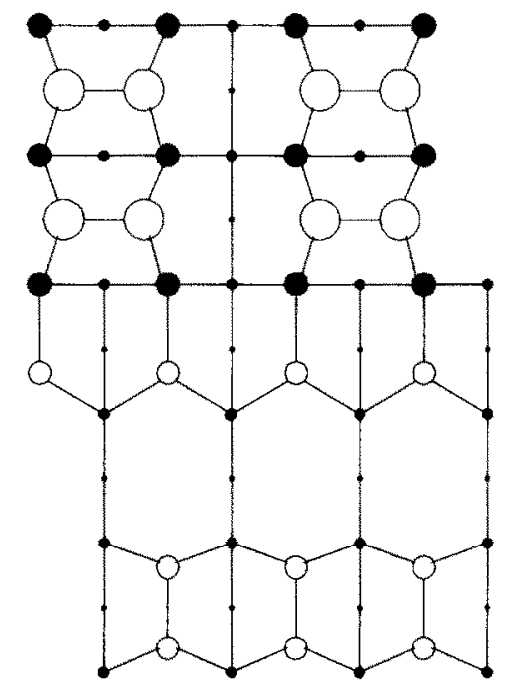

b

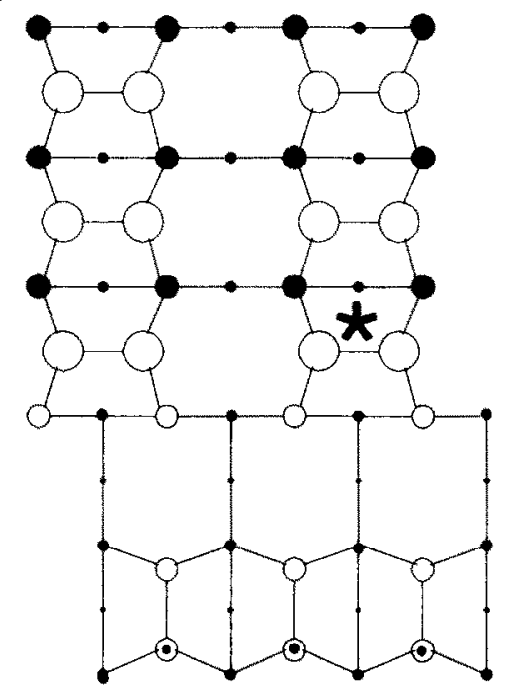

Fig. 1. (a, b) Top views of the rebonded (a) and the nonbonded (b) SB step respectively after Ref. [7]. Larger circles denote atoms on the upper terrace, open circles denote atoms with a dangling bond. A point denotes an upward buckled atom, i.e. out of the surface.

The former was found to be energetically more favourable with an energy difference of about $0.16 \mathrm{eV} / a_{0}$. The rebonded SB step, however, turned out to be less stable than the SA step. In accordance with these calculations, STM measurements of vicinal $\mathrm{Si}(001)$ reveal that the SB steps are much rougher than the SA steps. Moreover, the SB steps have almost exclusively the rebonded configuration. As a consequence of the $(1 \times 2)$ reconstruction, kinks are generally found on a $2 \times 2$ lattice. Hence, the kinks on vicinal Si $(001)$ have a minimum length of two dimers whereas kinks with a kink length of an odd number $\times a_{0}$ are only occasionally observed [6].

Using an ultra-high vacuum scanning tunnelling microscope we have investigated the vicinal $\mathrm{Ge}(001)$ surface with a miscut angle $\theta$ varying between $0.4^{\circ}$ and $5^{\circ}$ towards the [110] direction. In contrast to $\mathrm{Si}(001)$ we have found both the rebonded as well as the nonbonded configuration of the SB step. Moreover, there appeared to be a strong correlation between the kinks of the SB step.

From statistical analysis of our STM images the existence of three stable configurations of the SB step is deduced.

\section{Experimental}

All experiments were carried out in a stainless stecl cultra-high vacuum chamber with a base pressure of
$1.0 \times 10^{-10} \mathrm{mbar}$. The chamber contained an Omicron UHV STM, a cylindrical mirror analyzer Auger apparatus, an ion gun and facilities for gas handling. The $5 \times 10 \mathrm{~mm} \mathrm{Ge}(001)$ substrates were miscut towards the [011] direction by $0.4^{\circ}, 1.0^{\circ}, 2.0^{\circ}, 3.0^{\circ}$, and $5.0^{\circ}$, respectively.

Before loading in the UHV system they were ultrasonically cleaned in isopropyl alcohol. No further chemical treatments were carried out. The sample could be heated resistively in situ. A clean surface was obtained by cycles of sputtering ( $1000 \mathrm{eV}$ Ar ions, 2 $\mu \mathrm{A} / \mathrm{cm}^{2}, 30 \mathrm{~min}$, angle of incidence $20^{\circ}$ ) and annealing $(800 \pm 50 \mathrm{~K}, 20 \mathrm{~min})$. The temperature was measured with an infrared pyrometer.

After annealing, the samples were radiation quenched to room temperature by disconnecting the heating current. STM images were taken in the constant current mode with a sample bias between -1 and -2 $\mathrm{V}$ and a typical tunnelling current of $5 \mathrm{nA}$. The scan ranges varied between $70 \mathrm{~nm} \times 70 \mathrm{~nm}$ to $100 \mathrm{~nm} \times 100$ nm. $(0.08$ to $0.1 \mathrm{~nm} / \mathrm{pixel})$. The scanning direction was always oriented $45^{\circ}$ with respect to the dimer rows. Tips were made from tungsten wires by electrochemical etching in a $2 \mathrm{M}$ KOH solution. To obtain reasonable statistics several images were taken to achieve a total SB step length larger than $3600 a_{0}\left(a_{0} \approx 4 \AA\right)$ for each miscut angle. 

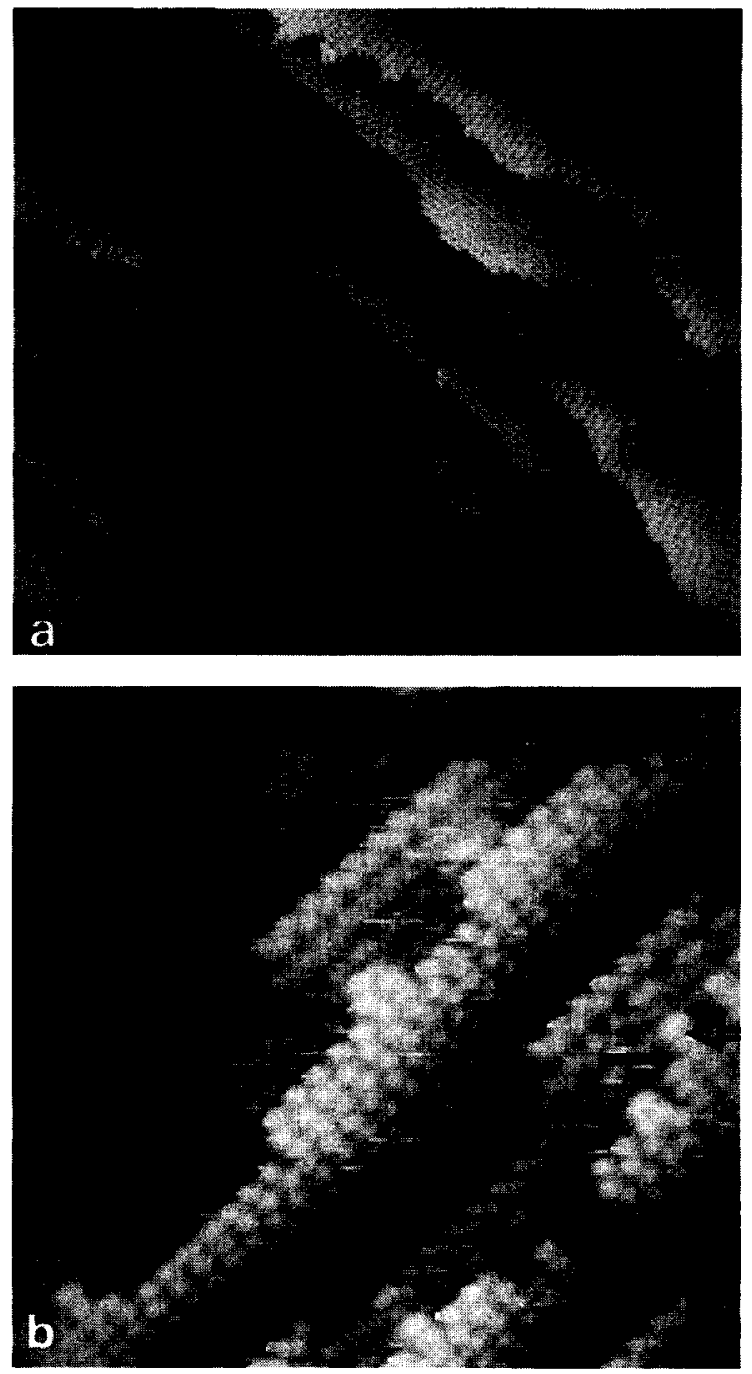

Fig. 2. (a) STM measurement of $\mathrm{Ge}(001)$ with a miscut angle $\theta$ of $1^{\circ}$. The arca is approximately $800 \times 800 \AA$. The surface is made up of alternately SA and SB steps. (b) STM measurement of Ge(001) with a miscut angle $\theta$ of $3^{\circ}$. The area is $190 \times 190 \AA$. Both the rebonded and nonbonded $S B$ steps are visible. The arrows indicate pieces of nonbonded SB steps. As can be seen, they are accompanied by antiferromagnetic coupling on the lower $(1 \times 2)$ terrace.

\section{Results and discussion}

In Figs. 2a and 2b typical STM images of $\mathrm{Ge}(001)$ with a miscut angle of $1^{\circ}$ and $3^{\circ}$ are shown. In marked contrast to $\mathrm{Si}(001)$ is the extremely low density of missing dimer defects. In accordance with $\mathrm{Si}(001)$ $[1,2,4]$, the SB step of the vicinal $\mathrm{Ge}(001)$ surface is much rougher, i.e., it exhibits many more thermally excited kinks compared with the SA step (see Fig. 2a). It must be noted that the straight SA steps on $\mathrm{Ge}(001)$ seem somewhat rougher as compared to the SA steps on vicinal $\mathrm{Si}(001)$. In marked contrast to silicon, however, the existence of both rebonded, as well as nonbonded, configurations of the SB step are found. Fig. $2 b$ shows a section of an SB step where both configurations are present. Another interesting feature is the fact that the non-bonded dimers at the SB step edge seem to induce buckling of the adjacent dimer row on the lower SA terrace. Remarkable to note is that all the dimers are buckled in the same direction, i.e. there seems to be a ferromagnetic-like coupling instead of an antiferromagnetic ordering of the dimers in a dimer row. The latter type of ordering has been observed by many others, whereas the former has never been reported before.

From Fig. 1b, the following observation about the dimers at the edge of the SB step can be made. Consider the step edge dimer marked " *". The two dimers that lie in front of it on the lower $(1 \times 2)$ terrace are buckled in such a way that the atoms closest to the SB step are the lower ones. Every edge dimer of the non-bonded SB step is accompanied by this buckling in all our experiments (see also Fig. 2b). This phenomenon can bc understood by taking into account the surface stress. The dimer bond induces considerable stress, for details see Ref. [8]. The stress is tensile in the direction of the dimer bond and compressive in the perpendicular direction. As a consequence a single domain surface can lower its energy by contraction in the direction of the dimer bond and expansion in the direction along the dimer rows. The buckling mentioned above may be understood qualitatively by the tendency of the $(2 \times 1)$

Table 1

Kink length distribution for a miscut angle $\theta$ of $2^{\circ}$; for $\theta=1^{\circ}$, a similar distribution is obtained

\begin{tabular}{lll}
\hline $\begin{array}{l}\text { Kink length } n \\
\left(a_{\mathrm{o}}\right)\end{array}$ & $\begin{array}{l}\text { Number of kinks } \\
\text { with a nonbonded } \\
\text { outward corner }\end{array}$ & $\begin{array}{l}\text { Number of kinks } \\
\text { with a rebonded } \\
\text { outward corner }\end{array}$ \\
\hline 0 & 211 & 548 \\
1 & 411 & 279 \\
2 & 355 & 126 \\
3 & 60 & 91 \\
4 & 46 & 22 \\
5 & 6 & 7 \\
6 & 5 & 4 \\
\hline
\end{tabular}




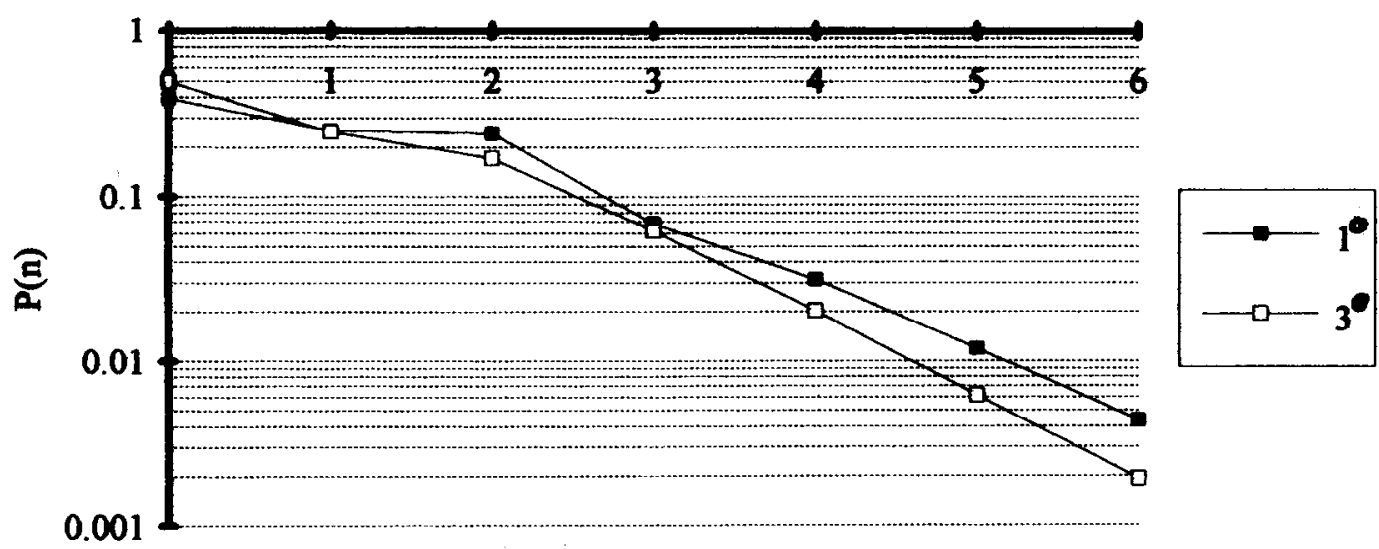

\section{kinklength ( $(00)$}

Fig. 3. Kink length distribution for a miscut angle $\theta$ of $1^{\circ}$ and $3^{\circ}$. It can be seen that a decrease of the terrace width leads to a suppression of long kinks.

dimer row of the upper terrace to expand itself and to shift the atoms on the lower $(1 \times 2)$ terrace away from the step.

\section{Kink length and kink separation distribution}

The STM images are used to chart the number of kinks of varying lengths, as well as the distribution of separations between kinks in STM images of the SA[9] and SB-type step. In this counting, the length $n$ of a kink refers to the number of dimer units between an inward and an outward corncr. For the SA step the separation $s$ between two kinks occurs in units of $a_{0}$ and $n$ in units of $2 a_{0}$ and, vice versa, for SB due to the $(2 \times 1)$ symmetry. The distribution of kink lengths $N(n)$ for the SB step is shown in Fig. 3 for miscut angles of $1^{\circ}$ and $3^{\circ}$.

Kink distributions of vicinal $\operatorname{Si}(001)$ and GaAs(001) [1-3] have been analyzed by assuming that the kinks are excited independently according to a Boltzmann distribution:

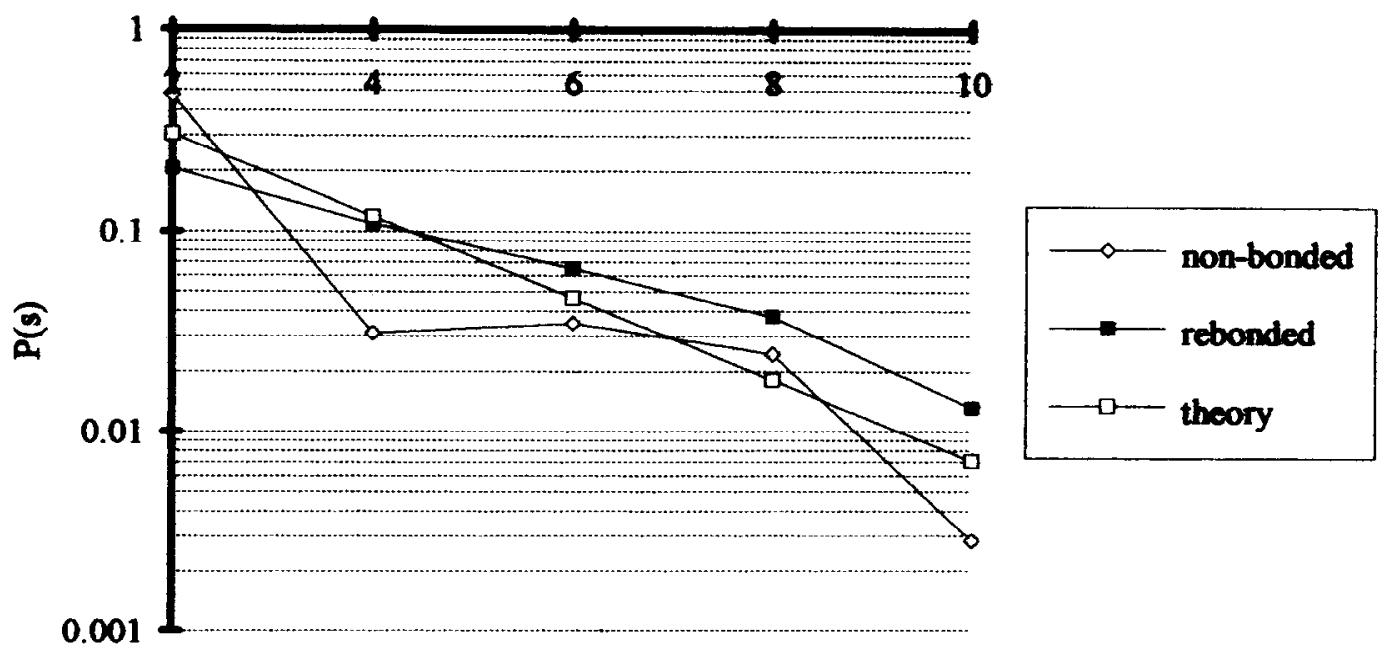

kinkseparation s (units 20 )

Fig. 4. Distribution of the kink separation for a sample with $\theta=1^{\circ}$. 
a

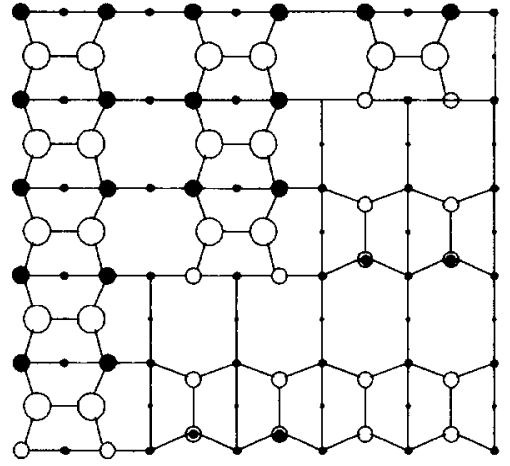

b

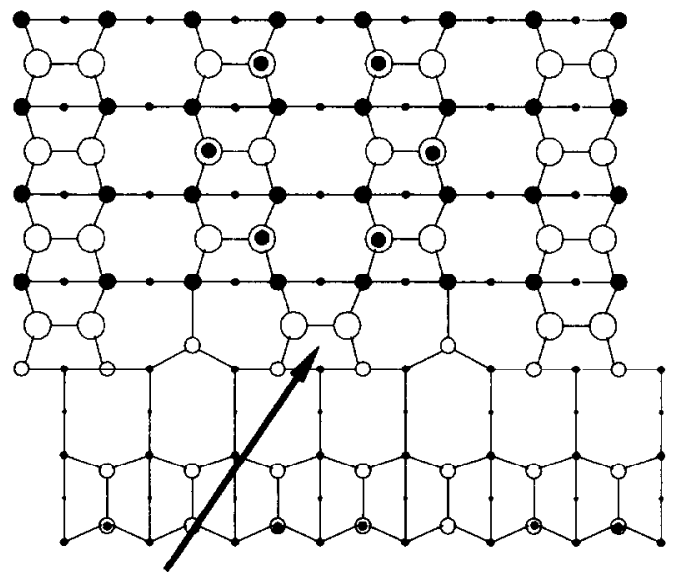

Fig. 5. Structures of the nonbonded step edges ( top views). An open circle denotes an atom with a dangling bond, a point denotes an upward buckled atom, i.e. out of the surface. (a) Structure for the step edge with an effective direction of $45^{\circ}$ with respect to the dimer rows. (b) Structure for the nonbonded step edge with a shifted dimer, which is indicated by the arrow.

$P(n) \propto \exp [-E(n) / k T]$,

$E(n)=n \epsilon_{\text {step }}+\epsilon_{\text {corner }}$

This approach is simple and neglects the suppression of long kinks when two step edges approach each other. We have considered the kink distribution by the transfer matrix method [10]. This method makes use of the partition sum and, therefore, it explicitly takes into account the suppressing of the excitation of kinks with decreasing terrace width, i.e. the entropic repulsion. Our calculations [11] showed however that the transfer matrix method cannot satisfactorily explain the kink distributions of Fig. 3.

More specifically it cannot explain the relatively low probability of $P\left(n=1 a_{0}\right)$ and high probability of $P\left(n=2 a_{0}\right)$. This is due to correlations between kinks, whereas it is essential for the transfer matrix method that the kinks are excited independently i.e. the presence of a kink should not influence the excitation at any other potential kink sites.

Further analysis of the kink distributions was made by differentiating between kinks with a non-bonded outward corner and a rebonded outward corner. Table 1 gives this distribution for a miscut angle of $2^{\circ}$. It is obvious that it deviates largely from the independent kink model which is applied to $\mathrm{Si}(001)$ and GaAs(001) [1-3] (formulas 1 and 2), especially in the case of kinks with a non-bonded outward corner.
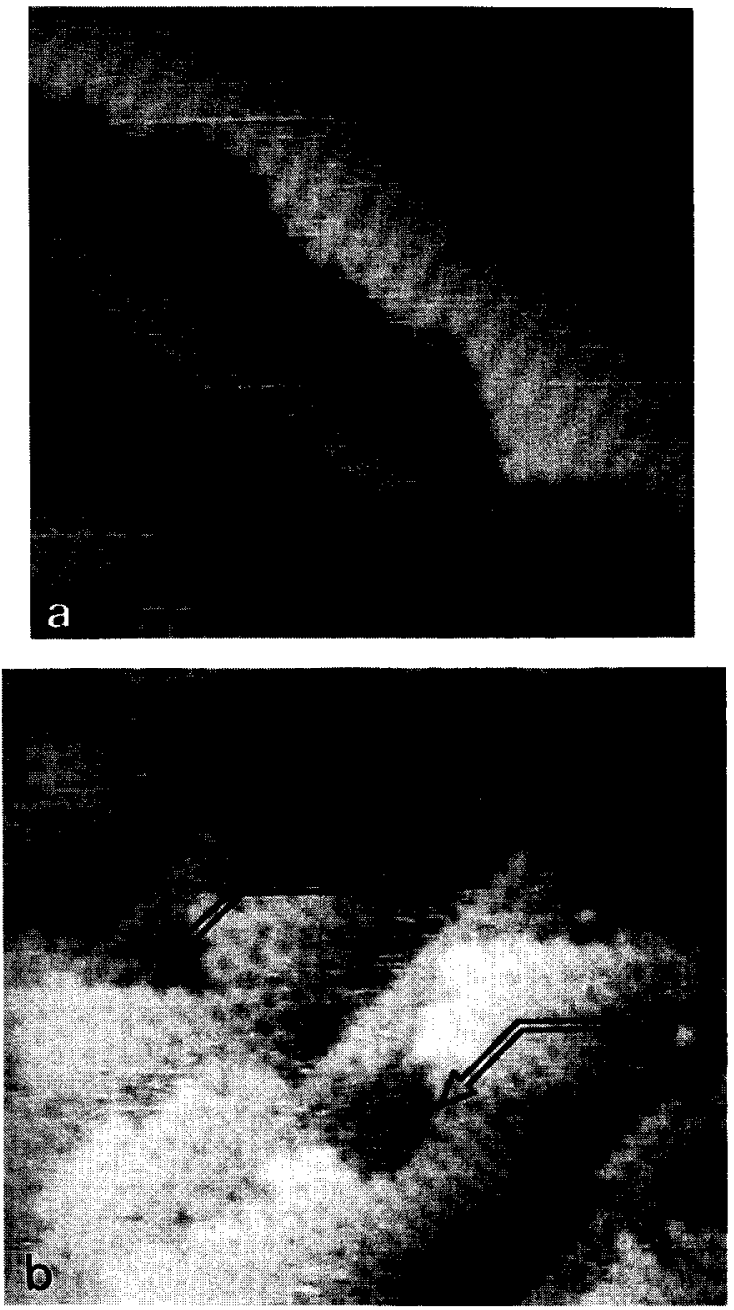

Fig. 6. (a, b) Examples of the nonbonded SB step with an effective direction of the step edge oriented at $45^{\circ}$ with respect to the dimer rows. The arrows point to the relevant edges. (a) Image of $200 \times 200$ $\AA, \theta=1^{\circ}$. (b) Image of $260 \times 260 \AA, \theta=2^{\circ}$. 

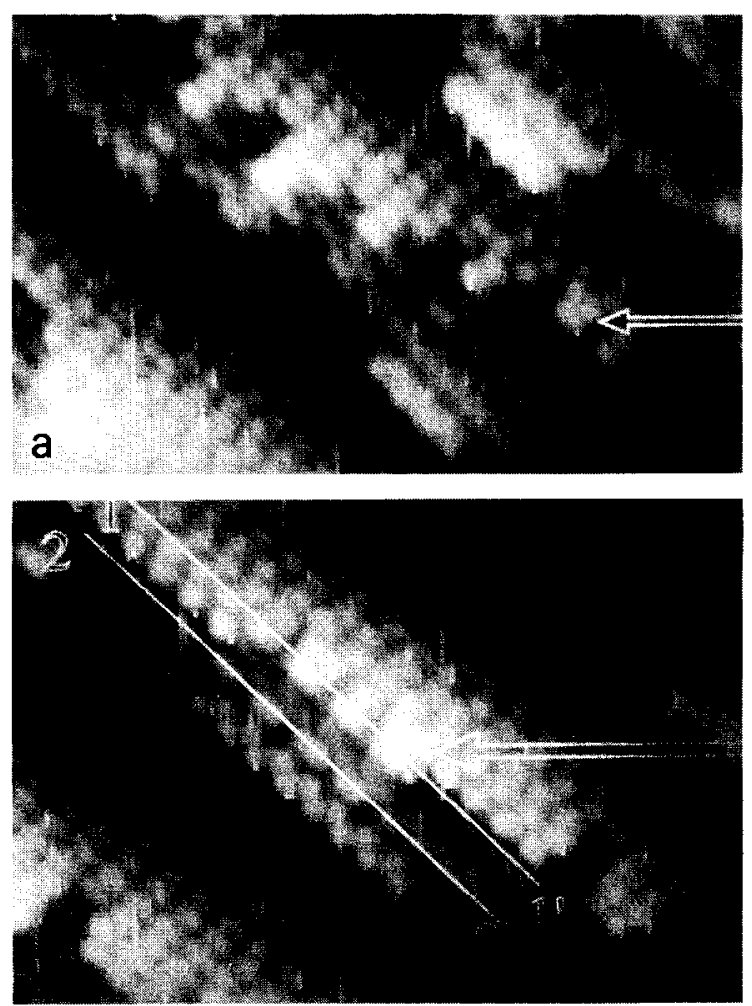

Fig. 7. (a, b) STM measurements of $\mathrm{Ge}(001)$ with a miscut angle of $3^{\circ}$. Tunnelling current is $4 \mathrm{nA}$, tunnelling voltage is $-2 \mathrm{~V}$ (filled states). The arrows point to the shifted dimer. (a) Scanwidth is $70 \times 40 \AA$. (b) Scanwidth is $70 \times 70 \AA$. The height profiles along line 1 and 2 are plotted in (c). As can be seen from line 1 , the height differences to the right and to the left of shifted dimer are approximately $1 \AA$. Line 2 shows that on the lower $(1 \times 2)$ terrace precisely the dimers that lic in front of the nonbonded step edge dimers have anti-ferromagnetic coupling.

For these kinks, a kink length 0 is even less frequently found than a kink length $1 a_{0}$ or $2 a_{0}$. From Table 1 it can also be concluded that a kink with a non-bonded dimer on the outer corner is energetically more favourable than a kink with a rebonded dimer on the outer corner.

The next issue we want to address is, whether or not kink-kink interactions exist. It is very important to make a division between nonbonded segments and rebonded segments in the SB-type step edges, as shown below. In the case of independent kinks the separation distribution $P(s)$ is simply represented by $0.5 \times P_{\mathrm{k}}\left(1-P_{\mathrm{k}}\right)^{s-1}[6]$, where $P_{\mathrm{k}}$ refers to the probability of finding a kink in an SB step edge segment. The factor 0.5 arises from the difference which is made between rebonded and nonbonded step edge dimers (the ratio between rebonded and nonbonded step edge dimers was almost 1 for a miscut angle $\theta$ of $1^{\circ}$ ). For the theoretical curve of Fig. $4, P_{\mathrm{k}}$ has been determined from the total number of kinks divided by the total number of potential kink sites. In the rebonded segments the kinks seem to occur, in excellent agreement with the rebonded SB step edges on vicinal $\mathrm{Si}(001)$, as totally independent, as the distribution is a straight line. Kinks in nonbonded step edge segments, however, certainly do not behave independently. Instead a preference for separation of $2 a_{0}$ is obviously present, whereas a separation of $4 a_{0}$ is clearly suppressed.

As mentioned above the configuration of the SB step of $\mathrm{Ge}(001)$ cannot be analyzed in the same way as $\mathrm{Si}(001)$ and $\mathrm{GaAs}(001)$. This is not only caused by the occurrence of both rebonded and non-bonded SB steps, but also by kink-kink interactions. However, from our data there arises a description of the SB step as a mixture of three stable configurations.

The first configuration is the rebonded SB step. It does not show kink-kink interaction. Moreover, kinks with a rebonded outward (see Table 1) corner follow roughly a Boltzmann distribution as illustrated by formula 1. From this we conclude that the rebonded SB step of $\mathrm{Ge}(001)$ behaves similarly to the SB step of $\mathrm{Si}(001)$.

For the nonbonded SB step we propose two stable configurations. The first is drawn schematically in Fig. 5a. It is characterized by an effective step edge directed $45^{\circ}$ with respect to the dimer rows and consists of, alternatingly, a piece of nonbonded SB step and a kink with length $2 a_{0}$. This configuration explains the high value for $P\left(s=2 a_{0}\right)$ in Fig. 4 and the distribution for $n=2 a_{0}$ in Table 1. Fig. 6 shows two examples. The $45^{\circ}$ direction suggests that samples with a miscut towards the [001] direction are assembled totally of steps with this configuration [12].

The second nonbonded configuration is drawn schematically in Fig. 6b and has so far not been reported. This configuration is observed for every miscut angle in all our measurements and is characterized by a single dimer that ends two dimer rows of the $(2 \times 1)$ terrace. Three arguments suggest that this shifted dimer is a dimer and not an effect caused by buckling. Firstly, height differences at both sides of the end dimer are 1.0 $\AA$. Height differences caused by buckling are, typically, $0.5 \AA$ or less. Secondly, as mentioned above, the dimer 


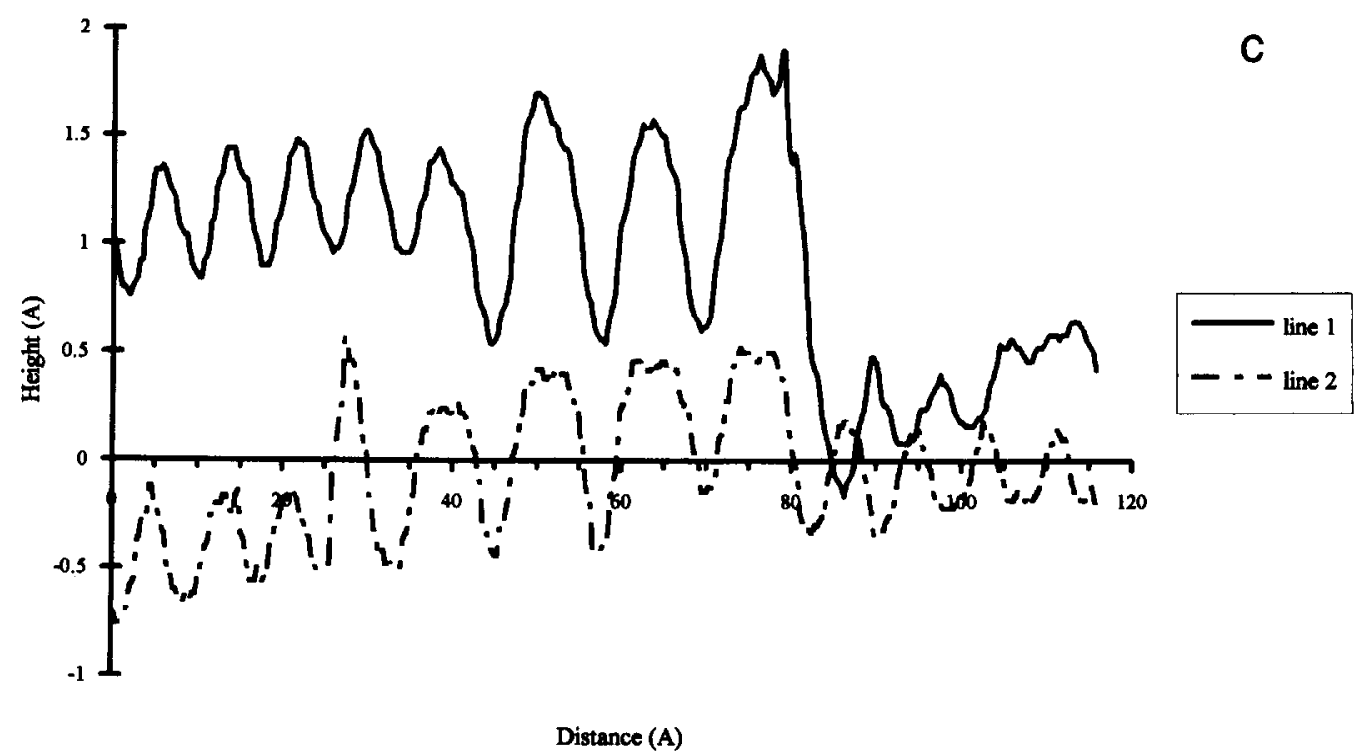

Fig. 7 (continued).

at the edge of the SB step is always accompanied by the same kind of buckling in all our measurcments. In the measurement of Fig. 7 exactly the dimers of the $(1 \times 2)$ terrace in front of the shifted dimer have this buckling. Finally, if the end dimer is not a dimer but consists of two upward buckled dimers, it would be a very rare and energetically unfavourable configuration as the neighbouring dimers of the same dimer rows have the same direction of buckling. The configuration we propose here is a mixture of the rebonded and the non-bonded configuration and has a periodicity of $6 a_{0}$ along the [110] direction. In the $P(s)$ distributions it accounts for the relatively high occurrence of $P\left(s=6 a_{0}\right)$ and $P\left(s=8 a_{0}\right)$. In fact all pieces of nonbonded SB steps longer than $2 a_{0}$ have this configuration.

\section{Conclusions}

We have studied, using STM, the Ge(001) surface with a small miscut angle towards the [011] direction. The surface was found to consist of, alternatingly, rough SB and smooth SA steps. In contrast to silicon both the non-bonded and the rebonded SB step were observed. From statistical analysis of the STM images we conclude that the rebonded SB step behaves similarly to the rebonded SB step of vicinal Si(001). For the non-bonded SB step two configurations are observed, one with a "shifted dimer", and one running in the [010] direction.

\section{References}

[1] B.S. Swartzentruber, Y.-W. Mo, R. Kariotis, M.G. Lagally and M.B. Webb, Phys. Rev. Lett. 65 (19900) 15, 1913.

[2] H.J.W. Zandvliet, H.B. Elswijk, E.J. van Loenen and D. Dijkkamp, Phys. Rev. B 45 (1992) 11, 5965.

[3] E.J. Heller, Z.Y. Zhang and M.G. Lagally, Phys. Rev. Lett. 71 (1993) 5, 743.

[4] B.S. Swartzentruber, N. Kitamura, M.G. Lagally and M.B. Webb, Phys. Rev. B 47 (1993) 20, 13432.

[5] J.E. Griffith, J.A. Kubby, P.E. Wierenga, R.S. Becker and J.S. Vickers, J. Vac. Sci. Technol. A 6 (1988) 493.

[6] R.J. Hamers, R.M. Tromp and J.E. Demuth, Phys. Rev. B. 34 (1986) 8, 5343.

[7] D.J. Chadi, Phys. Rev. Lett. 59 (1987) 15, 1691.

[8] M.C. Payne, N. Roberts, R.J. Needs, M. Needels and J.D. Joannopoulos, Surf. Sci. 211/212 (1989) 1.

[9] B. Kersten, H.J.W. Zandvliet and D.H.A. Blank, to be published.

[10] R. Kariotis and M.G. Lagally, Surf. Sci. 248 (1991) 295.

[11] Using the model of formulas 1 and 2 we obtained $\epsilon_{\mathrm{c}}$ ' $k T=-0.81$ and $\epsilon_{\text {step }} / k T=0.85$. Using $T=775 \mathrm{~K}$ (the anneal temperature) gave $\epsilon_{\mathrm{c}}=54 \mathrm{meV}$ and $\epsilon_{\text {step }}=73 \mathrm{meV}$. The transfer matrix method makes it possible to assign different energies to rebonded and nonbonded step segments and to kinks with a rebonded and nonbonded outward corner. None of the models used could however explain satisfactorily the distributions of Fig. 2 and Table 1.

[12] S. Mukherjee, E. Pelke and J. Tersoff, Phys. Rev. B 49 (1994) 3, 1919. 